\title{
Desmoid Type Fibromatosis of the Breast Masquerading as Breast Carcinoma: Value of Dynamic Magnetic Resonance Imaging and Its Correlation
}

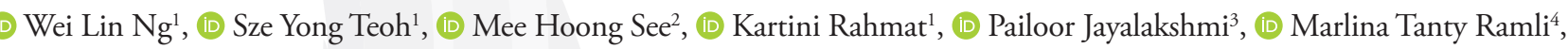
(1) Mei Sze Teh², (1) Anushya Vijayananthan ${ }^{1}$

${ }^{1}$ Department of Biomedical Imaging, University of Malaya, Kuala Lumpur, Malaysia

${ }^{2}$ Department of Surgery, University of Malaya, Kuala Lumpur, Malaysia

${ }^{3}$ Department of Pathology, University of Malaya, Kuala Lumpur, Malaysia (formerly)

${ }^{4}$ Department of Radiology, University Teknologi MARA, Selangor, Malaysia

\section{ABSTRACT}

Desmoid type fibromatosis of the breast is a rare stromal tumor that accounts for $<0.2 \%$ of all breast tumors. Bilateral and multicentric lesions are extremely rare, with only less than ten cases reported in the literature. Although benign, it is locally aggressive with frequent recurrence in up to almost one-third of the cases. We experienced our first case of bilateral multicentric breast fibromatosis in a 19-year-old woman, with a paternal aunt diagnosed with breast cancer at age 30, who presented to our institution with the chief complaint of retracted nipples for 1 year. The patient denied any history of trauma to her chest. Sonography showed suspicious bilateral hypoechoic masses. Magnetic resonance imaging (MRI) was performed for further evaluation because of the extensive involvement of both the breasts. This report aimed to illustrate the main clinical, radiological, and histopathological characteristics of this rare disease to increase awareness of this entity and discuss the role of MRI.

Keywords: Desmoid type fibromatosis, breast tumor, fibromatosis, magnetic resonance imaging, ultrasound

Cite this article as: Ng WL, Teoh SY, See MH, Rahmat K, Jayalakshmi P, Ramli MT, Teh MS, Vijayananthan A. Eur J Breast Health 2021; 17(2): 197-199

\section{Key Points}

- Desmoid type fibromatosis of the breast is a rare stromal tumour, however it is locally infiltrative with recurrence noted in up to almost one-third of the cases.

- MRI shows varying enhancement pattern and type 2 kinetic curve which mimics malignancy. An accurate evaluation of pectoralis major muscle involvement on MRI is important for surgical planning.

- The standard treatment of desmoid type fibromatosis is wide surgical resection with clear margins.

\section{Introduction}

Desmoid type fibromatosis of the breast is a rare stromal tumor that accounts for $<0.2 \%$ of all breast tumors. Although benign, this tumor is locally aggressive with frequent recurrence in up to almost one-third of the cases (1). Bilateral and multicentric lesions are extremely rare, found in only $4 \%$ of patients, with only less than ten cases reported in the literature $(1,2)$. Imaging features of aggressive fibromatosis can frequently mimic invasive breast cancer, resulting in exhaustive clinical and surgical workups.

\section{Case Presentation}

A 19-year-old woman, with a familial history of breast cancer (paternal aunt diagnosed with it at age 30), presented with bilateral breast lumps that were increasing in size and associated with nipple retraction for one year. Physical examination revealed bilateral retracted nipples. There was a $4 \mathrm{~cm} \times 5 \mathrm{~cm}$ firm, ill-defined, mobile retroareolar mass in her right breast and a $3 \mathrm{~cm} \times 3 \mathrm{~cm}$ firm, ill-defined, mobile mass in the upper outer quadrant of her left breast. There were no skin changes and fixation to the chest wall or skin. Axillary, supraclavicular, and infraclavicular lymph nodes were not palpable. Breast ultrasound showed bilateral ill-defined hypoechoic masses (two on the right, one on the left) with irregular margins and posterior shadowing (Figure 1). 
Initial core biopsy showed benign breast tissue. Because of imaginghistology discordance, magnetic resonance imaging (MRI) was performed for further evaluation. Contrast-enhanced MRI of both the breasts was performed using an MRI 3.0 T machine (Signa ${ }^{\circ} \mathrm{HDx}$ GE Healthcare, Wisconsin, USA). It demonstrated multiple irregular spiculated heterogeneously enhancing masses in the right breast extending from the nipple to the lower outer quadrant and upper half of the breast and collectively measuring $2.3 \mathrm{~cm} \times 5.1 \mathrm{~cm} \times 4.8 \mathrm{~cm}$ (Figure 2a, b). These lesions show corresponding hyperintense signals on T2/short tau inversion recovery imaging and hypointense signals on T1 imaging. The nipple-areolar complex was involved. There was no chest wall infiltration. Similar irregular spiculated enhancing

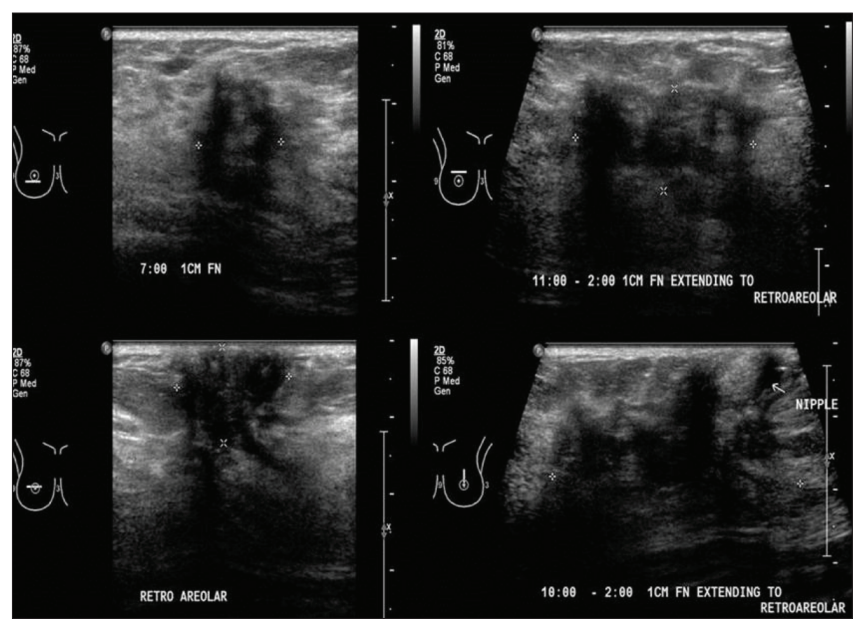

Figure 1. Irregular hypoechoic masses with posterior shadowing in the right seven o'clock position, right upper region, and retroareolar and left upper region. These suspicious bilateral heterogeneous breast lesions were categorized as BIRADS 4C

BIRADS: Breast Imaging Reporting and Database System score

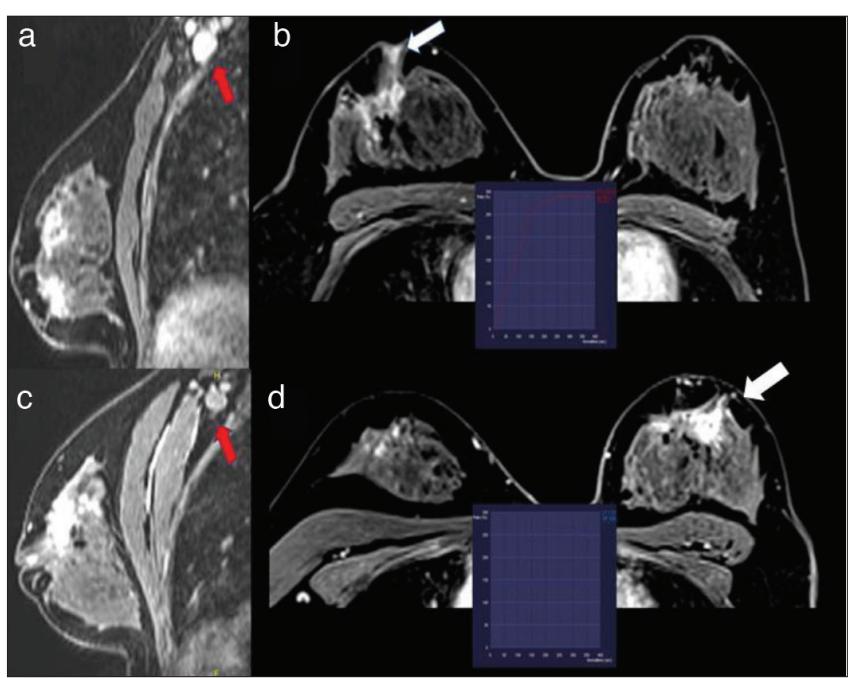

Figure 2. Dynamic post-contrast phase 3 magnetic resonance images in sagittal and axial views, of the right $(\mathbf{a}, \mathbf{b})$ and left $(\mathbf{c}, \mathbf{d})$ breasts demonstrate heterogeneously enhancing spiculated lesions in both the breasts. These lesions demonstrated type 2 kinetic curve pattern (inset). Multicentric lesions were seen on MRI with nipple-areolar complex involvement (white arrow). Abnormal lymph nodes are also seen in both axillae (red arrow)

MRI: Magnetic resonance imaging mass was seen in the upper half of the left breast, which measured approximately $2.0 \mathrm{~cm} \times 3.3 \mathrm{~cm} \times 4.1 \mathrm{~cm}$ (Figure 2c, d). Similarly, it extended into the nipple-areolar complex. Both the breast lesions demonstrated progressive enhancement that plateau on delayed phases consistent with a type 2 curve.

Ultrasound-guided hookwire excision biopsy was performed for three lesions (right at 12:00, left at 12:00, and left at 6:00), and result showed desmoid type fibromatosis.

A multidisciplinary team meeting convened owing to the rarity of this disease entity. The patient agreed for immediate skin-sparing mastectomy and reconstruction because of the local aggressiveness and frequent recurrence of this disease despite having no metastatic potential. The patient refused autologous or implant reconstruction and opted for staged lipofilling. The final histopathology showed proliferation of stellate to spindle-shaped cells. Nuclear positivity for $\beta$-catenin and $\mathrm{Ki}-67$ was $<1 \%$ (Figure 3).

\section{Discussion and Conclusion}

Desmoid type fibromatosis are rare, benign, and slow-growing fibroblastic neoplasm, common in women aged 22-49 years (1). It is locally aggressive with frequent recurrence and without distant metastasis. Associations with Gardner's syndrome, familial adenomatous polyposis, surgical trauma, or silicone breast implants have been reported. When associated with silicone implant, fibromatosis is thought to originate from the fibrous capsule of the implant $(1,2)$.

Clinically, fibromatosis of the breast is often mobile, non-tender firm masses, which sometimes appears to be adherent to the chest wall. Skin dimpling and nipple retraction have been observed, and these findings were similar to those in our patient (3).
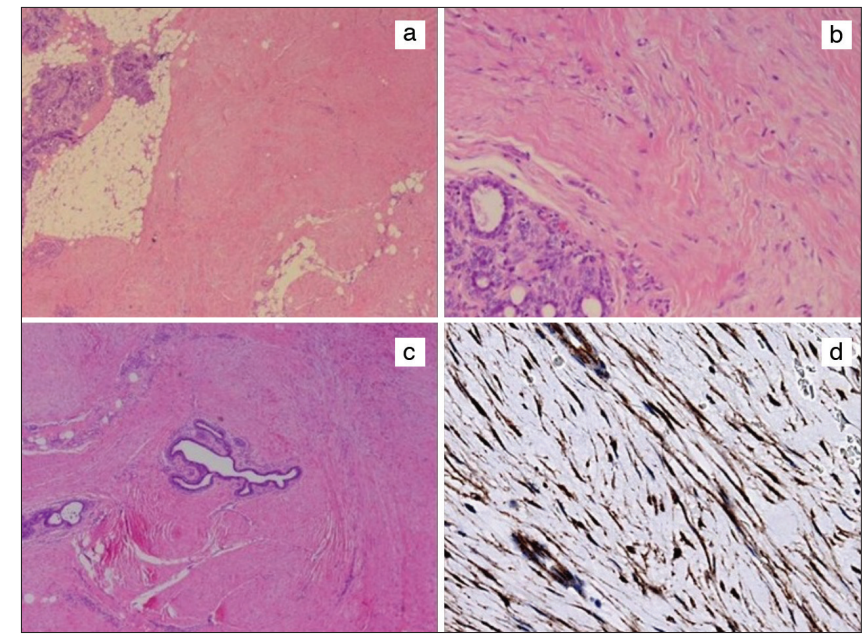

Figure 3. (a) Section of the breast showing the replacement of the breast tissue by fibrous tissue. Proliferation of stellate to spindleshaped cells in short intersecting fascicle. [hematoxylin \& eosin (H\&E) staining, under 40x magnification]. (b) The cells have elongated, spindle shapes and plump vesicular nuclei with occasional small nucleoli, pale acidophilic cytoplasm, and no distinct cell border (H\&E staining, under 40x magnification). (c) Entrapped benign breast ducts and acini, mild lymphocytic infiltration, and erythrocytes are seen surrounded by fibrous tissue (H\&E staining, under 100x magnification). (d) Immunohistochemistry: nuclear positivity for $\beta$-catenin; H\&E staining, under 400x magnification 
The reported cases of fibromatosis have been visible mammographically in only one-third of the cases. They are often irregularly shaped, noncalcified, high-density masses with spiculated margins that mimic breast cancer (1). However, mammography was not performed in our patient given her young age and expected dense breasts. Sonographically, the fibromatoses were solid, spiculated or microlobulated, hypoechoic masses with a thick echogenic rim and posterior attenuation. However, it can also present with benign features. Tethering of the Cooper's ligaments and involvement of the pectoralis muscle have been observed, indicating the locally aggressive nature of fibromatosis and accounting for the skin dimpling and nipple retraction $(1,2)$.

Although there is an extensive literature on MRI of musculoskeletal desmoid tumors, breast imaging features on MRI have been scarcely reported. Desmoid type fibromatosis appear as ill-defined, hypo to isointense masses on T1-weighted images and as heterogeneously hyperintense masses on T2-weighted images. These findings are similar to those in previous studies (4-7). On dynamic MRI, they often show a gradual enhancement thought to reflect the significant amount of collagenous tissue and myxoid change of the tumor (5). The enhancement pattern can vary. Certain cases of fibromatosis of the breast were reported to have rapid enhancement and washout on dynamic MRI (type 2 curve), mimicking that of an invasive carcinoma. In such cases, the lack of peripheral ring enhancement, typical for breast cancers, can be a differentiating feature (8). MRI also provides an accurate evaluation of the involvement of the pectoralis major muscle, which is important for surgical planning $(5,8)$.

Grossly, the appearance of desmoid type fibromatosis can vary from being well-circumscribed nodular lesions to irregular infiltrative lesions. Histologically, the hallmark of a desmoid type fibromatosis is the presence of non-encapsulated bland-looking spindle cells organized into long sweeping and intersecting fascicles with fingerlike extensions at the periphery of the lesion into adjacent the breast parenchyma and adipose tissue (9). In desmoid type fibromatosis, the overall cellularity is low to moderate with no cytologic pleomorphism or increase in mitotic activity, which is important to distinguish it from metaplastic fibrosarcoma. In case of immunohistochemistry, positivity for actin and vimentin is useful for the diagnosis of desmoid type fibromatosis. Desmin is rarely positive, whereas $\mathrm{S} 100$ and CD34 are usually negative. $\beta$-catenin nuclear staining is also an option for diagnosis, but it may be only focally positive. Meanwhile, cytokeratin staining is helpful in ruling out a carcinoma $(1,9)$.

Given the local aggressiveness and frequent recurrence, the standard treatment of desmoid type fibromatosis involves a wide surgical resection with clear margins (10). Povoski et al. (11) reported performing repeated excision in a patient because of positive margin from primary surgery. Mastectomy was performed for our patient because of the multicentric lesions and nipple-areolar complex involvement of the disease. Clear margins were obtained.

Desmoid type fibromatosis of the breast is an unusual but distinct entity. Given the patient's young age and familial history of breast cancer, MRI of the breasts is preferred over mammogram since the patient has denser breasts. All clinicians should be aware of this disease entity. Any discordance in clinical, radiological, and pathological assessment for any of the breast cancer symptoms must be further discussed and investigated. Although desmoid tumors have no metastatic potential, its local aggressiveness may be devastating if its treatment is delayed.

Informed Consent: Informed consent was obtained from the patient.

Peer-review: Externally peer-reviewed.

\section{Author Contributions}

Concept: A.V.; M.H.S.; Design: A.V.; M.T.R.; Supervision: A.V; K.R.; Resources: P.J.; M.S.T.; Materials: S.Y.T.; Data Collection and/or Processing: S.Y.T.; Analysis and/or Interpretation: S.Y.T.; W.L.N.; Literature Search: S.Y.T.; Writing Manuscript: S.Y.T.; Critical Review: W.L.N.

Conflict of Interest: The authors have no conflicts of interest to declare.

Financial Disclosure: The authors declared that this study has received no financial support.

\section{References}

1. Glazebrook KN, Reynolds CA. Mammary fibromatosis. AJR Am J Roentgenol 2009; 193: 856-860. (PMID: 19696302) [CrossRef]

2. Wongmaneerung P, Somwangprasert A, Watcharachan K, Ditsatham C. Bilateral desmoid tumor of the breast: case seriesand literature review. Int Med Case Rep J 2016; 9: 247-251. (PMID: 27578999) [CrossRef]

3. Erguvan-Dogan B, Dempsey PJ, Ayyar G, Gilcrease MZ. Primary desmoid tumor (extraabdominal fibromatosis) of the breast. Am J Roentgenol 2005; 185: 488-489. (PMID: 30233754) [CrossRef]

4. Steadman L, Crook S. Fibromatosis arising from the pectoralis major muscle mimicking breast cancer. Radiol Case Rep 2018; 13: 1174-1178. (PMID: 30233754) [CrossRef]

5. Linda A, Londero V, Bazzocchi M, Zuiani C. Desmoid tumor of the breast: radiologic appearance with a focus on its magnetic resonance features. Breast J 2008; 14: 106-107. (PMID: 18186874) [CrossRef]

6. Grimaldi MC, Trentin C, Gullo RL, Cassano E. Fibromatosis of the breast mimicking cancer: a case report. Radiol Case Rep 2018; 13: 1-5. (PMID: 29487630) [CrossRef]

7. Nakazono T, Satoh T, Hamamoto T, Kudo S. Dynamic MRI of fibromatosis of the breast. Am J Roentgenol 2003; 181: 1718-1719. (PMID: 14627606) [CrossRef]

8. Mesurolle B, Leconte I, Fellah L, Feger C, Nakazono T, Kudo S. Dynamic breast MRI in recurrent fibromatosis. AJR Am J of Roentgenol 2005; 184: 696-697. (PMID: 15671404) [CrossRef]

9. Aitken SJ, Presneau N, Kalimuthu S, Dileo P, Berisha F, Tirabosco R, et al. Next-generation sequencing is highly sensitive for the detection of beta-catenin mutations in desmoid-type fibromatoses. Virchows Archiv 2015; 467: 203-210. (PMID: 25838078) [CrossRef]

10. Benej R, Mečiarová I, Pohlodek K. Desmoid-type fibromatosis of the breast: A report of 2 cases. Oncol Lett 2017; 14: 1433-1438. (PMID: 28789360) [CrossRef]

11. Povoski SP, Marsh WL, Spigos DG, Abbas AE, Buchele BA. Management of a patient with multiple recurrences of fibromatosis (desmoid tumor) of the breast involving the chest wall musculature. World J Surg Oncol 2006; 4: 32. (PMID: 16768799) [CrossRef] 